\title{
HIV and Reproduction: Fertility, Contraception, and Preconception Issues and Interventions
}

\author{
Jean R. Anderson, ${ }^{1}$ Deborah Cohan, ${ }^{2}$ and Susan Cu-Uvin ${ }^{3}$ \\ ${ }^{1}$ Department of Gynecology and Obstetrics, Johns Hopkins Medical Institutions, Baltimore, MD 21287, USA \\ ${ }^{2}$ Department of Obstetrics and Gynecology, University of California at San Francisco, San Francisco, CA, USA \\ ${ }^{3}$ Department of Obstetrics and Gynecology, Brown University, Providence, RI 02906, USA
}

Correspondence should be addressed to Jean R. Anderson, janders@jhmi.edu

Received 23 December 2012; Accepted 23 December 2012

Copyright (c) 2012 Jean R. Anderson et al. This is an open access article distributed under the Creative Commons Attribution License, which permits unrestricted use, distribution, and reproduction in any medium, provided the original work is properly cited.

The cumulative advances in HIV care and treatment and in prevention of perinatal transmission over the past decade and a half have led not only to significant reductions in mortality and morbidity for women living with HIV and dramatic reductions in new infections in infants, but also to a sea change in the lens through which HIV-infected women view their lives and possibilities. In this context the $150 \%$ increase in live births to HIV-infected women in the era of effective combination antiretroviral therapy [1] can be seen as a sign of success. Somewhat belatedly HIV and women's health providers have recognized the critical need to address issues related to the fertility of women with HIV proactively and without judgment.

Although a substantial number of HIV-infected individuals express the intention to have children, unintended pregnancy rates remain high, and there continue to be questions regarding the safety of contraceptive methods, particularly hormonal contraceptives, in the setting of HIV. The more recent developments in biomedical prevention, including preexposure prophylaxis (PrEP) and antiretroviral therapy (ART) as prevention, offer new opportunities for safe conception with HIV serodiscordant couples.

This special issue contains 13 papers, which address a range of reproductive issues relevant to fertility in the setting of HIV in both resource-rich and resource-limited settings. Although not exhaustive, they demonstrate both the diversity of issues that need to be considered and the accumulation of knowledge in this area, now over 30 years into the HIV/AIDS pandemic. We thank the authors for their excellent contributions and our reviewers for their thoughtful reading and suggestions that have strengthened this offering.

In the paper "Pharmacokinetic interactions between the hormonal emergency contraception, levonorgestrel (plan B), and Efavirenz" M. L. Carten et al. examine the area under the curve concentration of single-dose levonorgestrel (LNG) alone and after 14 days of efavirenz (EFV) exposure in 21 women. They report that EFV significantly reduced LNG exposure, with implications for the efficacy of LNG as a widely used emergency contraception method and the potential need for higher doses in the setting of EFV-based ART.

S. Mark et al. in "HIV mother-to-child transmission, mode of delivery, and duration of rupture of membranes: experience in the current era" report a retrospective series of $210 \mathrm{HIV}$ infected pregnant women on effective combination ART with viral suppression $<1000$ copies $/ \mathrm{mL}(<50$ copies $/ \mathrm{mL}$ in $80 \%)$ to examine the effect of duration of membrane rupture on perinatal transmission in the setting of viral suppression. Although almost half of women were delivered by cesarean delivery, $28 \%$ had membrane rupture for four hours or longer and $16 \%$ rate had preterm birth; there were no cases of perinatal transmission, giving further support for the effectiveness of ART and viral suppression in the setting of membrane rupture for more prolonged periods of time.

"Reproductive healthcare needs and desires in a cohort of HIV-positive women" by M. L. Badell et al. reports survey results examining contraceptive use, desires, and knowledge along with future fertility desires and sterilization regret in $127 \mathrm{HIV}$-infected women receiving care at an urban 
infectious disease clinic. Approximately one-third desired future fertility, including $18 \%$ of women who had undergone sterilization. Less than $1 \%$ of women used a long acting reversible method of contraception, and only one-half of those sexually active had discussed contraception with their providers in the previous year, identifying important gaps in reproductive health care for these women.

Because of concerns about increased teratogenic risk with efavirenz exposure in early pregnancy, EFV use has historically been discouraged in women planning pregnancy or not using effective contraception; however, EFV-based regimens are widely used for treatment because of their potency and relative simplicity and are a primary treatment regimen recommended by WHO in limited resource setting. S. Schwartz et al. in "Efavirenz conceptions and regimen management in a prospective cohort of women on antiretroviral therapy" report on a prospective cohort of over 800 HIVinfected South African women on ART, finding high rates of pregnancy on EFV and inconsistencies in management of women trying to conceive while on EFV, highlighting the need for clearer guidelines around the issue of fertility in women on EFV-based regimens.

J. Firth et al. in "the changing face of HIV in pregnancy in Rhode Island 2004-2009" call attention to the changing demographics of HIV in pregnancy being seen in many US locations, with an increasing proportion of foreign-born HIV-infected pregnant women, as well as an increase in repeat pregnancies after learning of an HIV diagnosis. These and other changes are important to track, as they will affect the specific and evolving needs of HIV-infected women in pregnancy.

In "Incidence of pregnancy after initiation of antiretroviral therapy in South Africa: a retrospective clinical cohort analysis" D. Westreich and colleagues evaluated 5,996 women who experienced 727 pregnancies for an overall pregnancy rate of 5.2 per 100 person-years; after 6 years, the cumulative incidence of pregnancy was $52.2 \%$ among women ages $18-25$ at ART initiation. Lower CD4 cell counts and poor adherence to ART were associated with lower pregnancy rates. This paper reinforces other studies that suggest that ART may restore or improve fertility, with significant implications for contraceptive counseling and access.

E. Aaron et al. address the relationship between smallfor-gestational-age (SGA) infants and HIV in "Small for gestational age births in pregnant women with HIV, due to severity of HIV disease, not antiretroviral therapy." In their urban cohort of 183 HIV-infected women, rate of SGA was high and most notably associated with smoking. Importantly, ART, whether NNRTI or PI based, was not associated with increased risk of SGA, but more advanced $\mathrm{HIV}$, as reflected by CD 4 count $<200$ cells $/ \mathrm{mm}^{3}$, was.

The intrauterine contraceptive device (IUCD) is an effective long-acting and reversible method of contraception, and both the Cu-IUCD and levonorgestrel-containing IUCD can be safely initiated or continued in women with HIV/AIDS who are clinically doing well on ART [2]. However, C. S. Todd et al. found that just over one-third of HIV-infected women attending a primary health care clinic in South Africa were aware of the IUCD in "Aawareness and interest in intrauterine contraceptive device use among HIV-positive women in Cape Town, South Africa." However, after learning about this method, $86 \%$ of women expressed interest in the IUCD, pointing to the need not only for access to this method but also access to information.

A qualitative analysis of $30 \mathrm{HIV}$-infected Kenyan women accessing HIV treatment, "Fertility intentions and interest in integrated family planning services among women living with HIV in Nyanza Province, Kenya: a qualitative study" by E. K. Harrington et al. found a significant expressed need for contraception; however, only one-third were using a modern method of contraception other than condoms. Women expressed a strong preference for integrated HIV and family planning services.

O. Mmeje et al. in "Evaluating safer conception options for HIV-serodiscordant couples (HIV-infected female/HIVuninfected male): a closer look at vaginal insemination" review safer conception strategies for serodiscordant couples in which the woman is HIV-infected. They focus on vaginal insemination of partner's semen during the fertile period of the woman's cycle, coupled with $100 \%$ condom use during sexual contact, as a safe and effective method to achieve conception.

J. A. Robinson et al. present a detailed and extensive review of a topic that is of significant interest and importance with implications for both safety and effectiveness of concomitant use of ART and hormonal contraception in "Contraception for the HIV-positive woman: a review of interactions between hormonal contraception and antiretroviral therapy."

Serodiscordant couples were also the focus of L. T. Matthews et al.'s paper "Reproductive counseling by clinic healthcare workers in Durban, South Africa: perspectives from HIV-infected men and women reporting serodiscordant partners." In in-depth interviews with $30 \mathrm{HIV}$-infected women and 20 HIV-infected men, all in serodiscordant relationships, there was interest in safe conception advice and willingness to discuss this with providers, but these conversations seldom took place, highlighting the need for providers to be more proactive in assessing and addressing this need.

Finally, in "Preconception and contraceptive care for women living with HIV," M. J. Hoyt et al. review the rationale and the content of preconception counseling and care for HIV-infected women, advocating this as an essential component of primary health care services for all women living with HIV and with the capacity for childbearing.

In totality, these 13 papers illustrate the astounding diversity of issues that are of interest and importance in the area of fertility, pregnancy, and reproductive health for women living with HIV. Three decades into the HIV/AIDS pandemic, we welcome this focus on women and issues of critical importance to them both for care and for quality of life.
Jean R. Anderson

Deborah Cohan

Susan Cu-Uvin 


\section{References}

[1] A. Sharma, J. G. Feldman, E. T. Golub et al., "Live birth patterns among human immunodeficiency virus-infected women before and after the availability of highly active antiretroviral therapy," American Journal of Obstetrics and Gynecology, vol. 196, no. 6, pp. e541-e546, 2007.

[2] CDC. U. S., "Medical eligibility criteria for contraceptive use," MMWR. Recommendations and Reports, vol. 59, no. RR-4, pp. $1-86,2010$. 


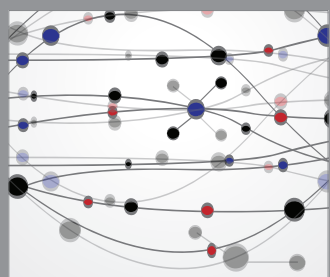

The Scientific World Journal
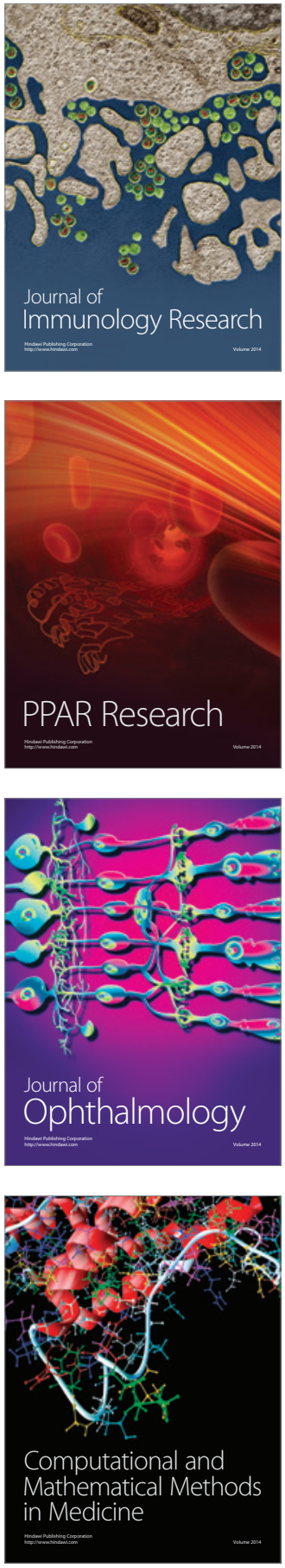

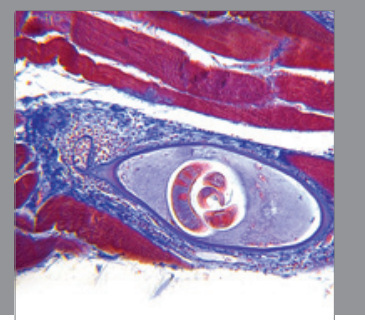

Gastroenterology

Research and Practice
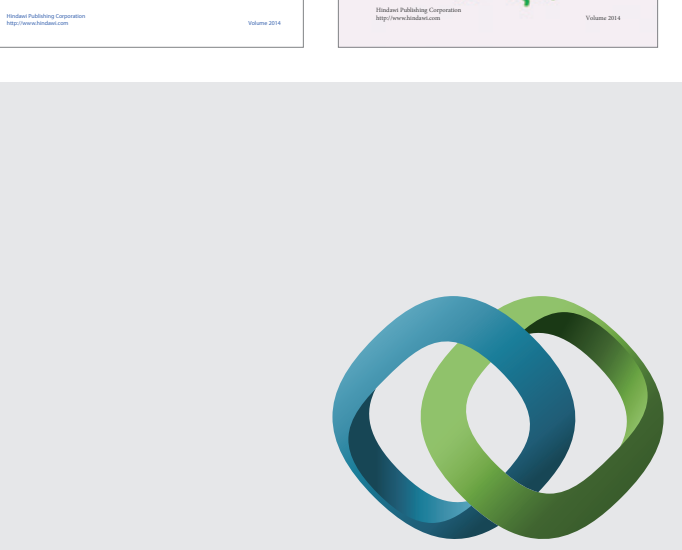

\section{Hindawi}

Submit your manuscripts at

http://www.hindawi.com
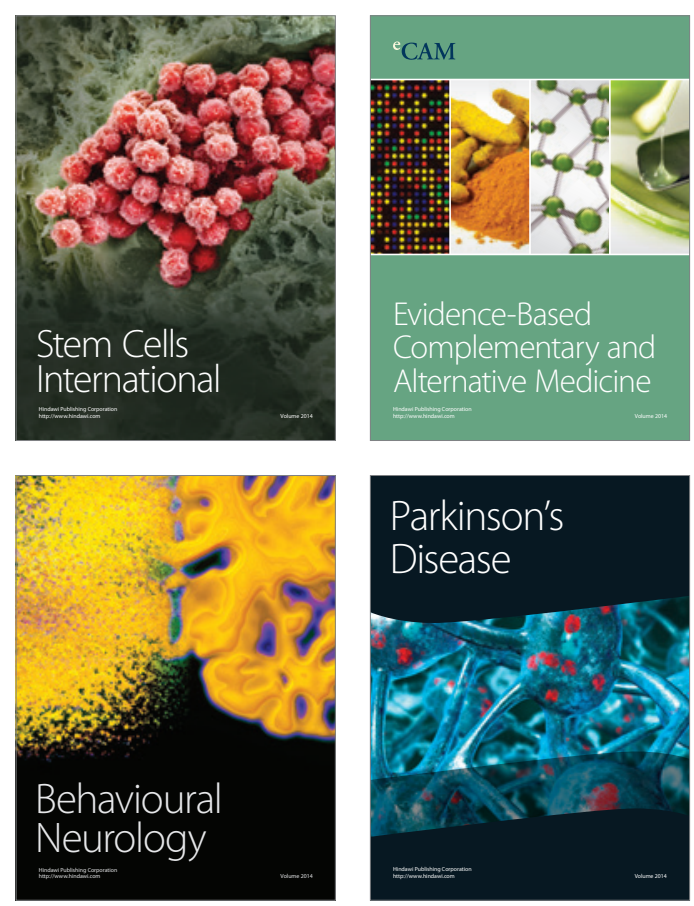

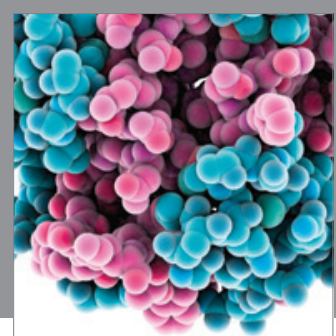

Journal of
Diabetes Research

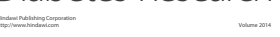

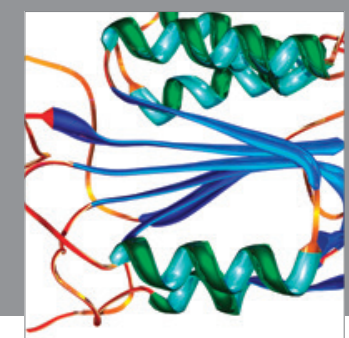

Disease Markers
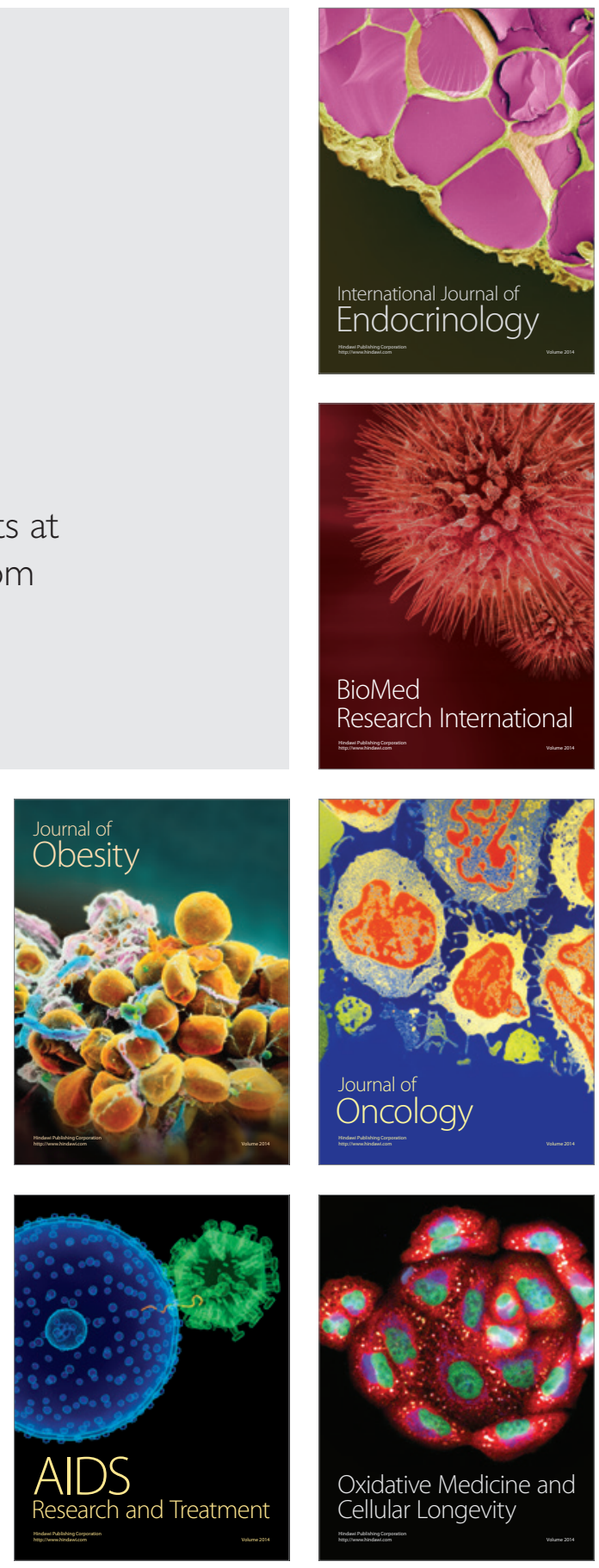